\title{
Obtaining the VLPs from Recombinant Core Protein of Hepatitis C Virus (HCV)
}

Anastasia Pechelyulko $^{1}$, Y Tarakanova ${ }^{1}$, Olga Sokolova ${ }^{2}$, Y Massino $^{1}$, Alexander Dmitriev ${ }^{2}$, O Segal $^{1}$ and Mary Lichutina ${ }^{1}$

${ }^{1}$ Mechnikov Scientific Research Institute of Vaccines and Sera, Moscow, Moskva, Russia, ${ }^{2}$ Lomonosov Moscow State University, Faculty of Biology, Moscow, Moskva, Russia

Hepatitis $\mathrm{C}$ is a worldwide recognized social disease. The causative agent of the disease - the hepatitis $\mathrm{C}$ virus $(\mathrm{HCV})$-can induce both acute and chronic hepatitis with varying degrees of severity. Diagnosis of $\mathrm{HCV}$ infection requires both a positive HCV antibody screen and confirmatory nucleic acid testing (NAT). Antibody assays of the latest generation also include the detection of structural virus protein, namely HCV core antigen $(\mathrm{HCVcAg})$, to increase test sensitivity and reduce the diagnostic window period. Testing for $\mathrm{HCVcAg}$ is also considered as a potential alternative to NAT [1]. In addition, HCVcAg could be of interest from the viewpoint of vaccine design. It is important that HCVcAg has the capacity to form virus-like particles (VLPs), which are considered as valuable tools in modern vaccine technology $[2,3]$.

The aim of the present work is to develop and optimize the procedure for obtaining VLPs, assembled from HCVcAg, expressed in Pichia pastoris. The core protein (CP) was precipitated with ammonium sulfate at saturation of 0.1, and the renaturation was performed by gel filtration on Sephadex G-25 under reducing conditions. VLPs were generated as a result of a gel filtration on Sephadex G-25 in the absence of a reducing agent. Protein purity and specificity were evaluated by SDS-PAGE and immunoblotting analysis, the molecular mass of VLPs and their relative quantity in protein preparation were measured by HPLC. The validation of the particle size was accomplished by negative stain EM. Micrographs were acquired using an analytical transmission electron microscope Jem-2100(Jeol, Japan) equipped with a $2 \mathrm{~K} \times 2 \mathrm{~K}$ CCD camera Ultrascan 1000XP (Gatan, USA). The microscope was operated at $200 \mathrm{kV}$ in a low dose mode, with a magnification of $\times 20000$ and a defocus of $0.5-1.9 \mu \mathrm{m}$.

VLP average sizes were determined on TEM images after classification in EMAN2.0 [4] (Fig. 1). The average VLP size was $60 \mathrm{~nm}$, which is consistent with the size of the native capsids [5].

As a result, we developed a simple procedure that allowed us to obtain recombinant HVCcAg preparation (with $\geq 90 \%$ purity) in the form of VLPs, for further use in diagnostic test systems and vaccine engineering $[6]$. 


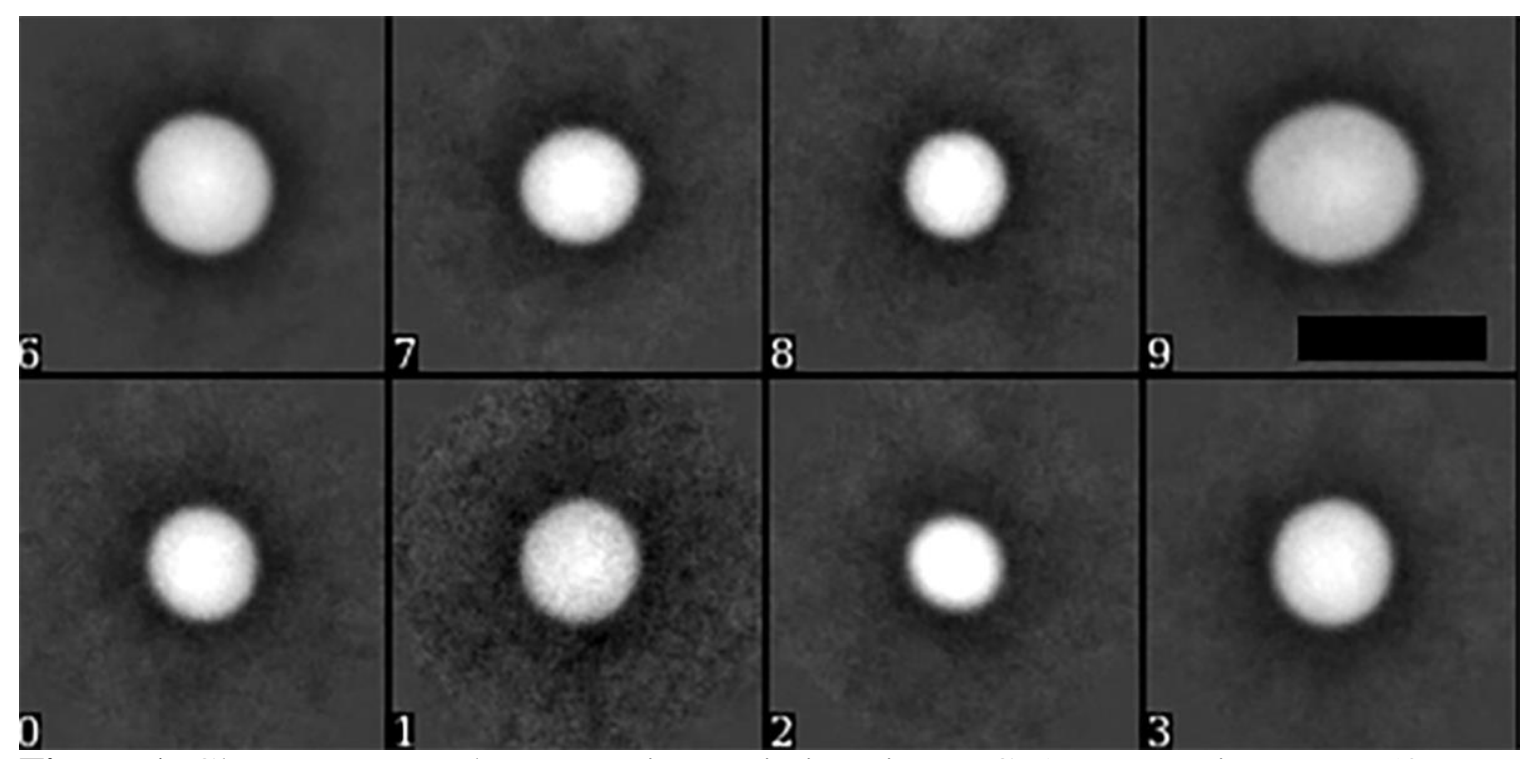

Figure 1. Class-averages, demonstrating variations in HVCcAg VLPs size. Bar - $50 \mathrm{~nm}$.

\section{References}

[1] WHO guidelines on hepatitis B and C testing (2017)

[2] N. Acosta-Rivero et al. BBRC 325 (2004) 68-74

[3] B. Bellier and D. Klatzmann Expert Rev. Vaccines 12(2), 143-154 (2013)

[4] G. Tang et al. J Struct Biol. 157, (2007) 38-46.

[5] M.T. Catanese et al. Proc Natl Acad Sci U S A, 110 (2013), pp. 9505-9510

[6] Electron microscopy was performed using the Unique equipment setup "3D-EMC" of Moscow State University (supported by the Ministry of science and higher education of the Russian Federation, unique identifier RFMEFI61919X0014). 